\title{
Las potencias regionales como protagonistas del sistema político internacional: cooperación y diálogo en el Foro BRICS*
}

\author{
Daniel Efrén Morales RUVALCABA \\ Departamento de Estudios Ibéricos y Latinoamericanos \\ Universidad de Guadalajara \\ demgdl@gmail.com \\ Alberto RochA VALENCIA \\ Departamento de Estudios Ibéricos y Latinoamericanos \\ Universidad de Guadalajara \\ albertorochav@yahoo.com.mx \\ Elizabeth VARGAS GARCÍA \\ Departamento de Estudios Ibéricos y Latinoamericanos \\ Universidad de Guadalajara elivargar@hotmail.com
}

Recibido: 17-07-2013

Aceptado: 17-02-2014

\section{RESUMEN}

Las potencias regionales comenzaron a jugar roles geoeconómicos y geopolíticos destacados en el Sistema de Relaciones Internacionales desde los años 1990, pero se han manifestado con mayor notoriedad solamente desde los años 2000, cuando se hizo evidente que habían emprendido un proceso de ascenso en la pirámide de poder político mundial. Desde entonces, estas potencias regionales comenzaron a aproximarse, converger y cooperar, dando paso a la formación de un Foro Internacional: el BRICS. Muy pronto, este Foro, se convirtió en un actor internacional colectivo de primer orden, juntamente con el G-20 y en competencia con el G-7. Este artículo aborda la naturaleza constitutiva (semiperiférica) y la posición estructural de potencias regionales ascendentes de los Estados que constituyen el Foro BRICS. Asimismo, se analiza cada una de sus Cumbres y la geopolítica que implementan conjuntamente. Según nuestro análisis, todo indica que los integrantes del Foro están llamados a jugar roles protagónicos en el proceso de cambio y transformación del orden político internacional en el siglo XXI.

Palabras clave: Sistema Político Internacional; BRICS; potencias regionales; geopolítica; semiperiferia.

\footnotetext{
* Destacamos y agradecemos el quehacer de Tania Duran González y Mónica Apango Partida por su apoyo en las labores de investigación y sistematización de información durante la realización de este documento.
} 


\title{
Regional powers as protagonists of the International Political System: Cooperation and dialogue within the BRIC Forum
}

\begin{abstract}
While regional powers started to play relevant geo-economic and geopolitical roles in the international relations system in the 1990s, it was not until the 2000s when they reached out even more outstanding positions. The fact that they had begun an ascending process in the pyramid of world political power became simply obvious. Since then, these regional powers started to come closer, to converge and cooperate, thereby moving towards the construction of an international forum: the BRIC Forum. Very early in this process, this Forum became a first-class collective international actor, together with the G-20 and in competition with the G-7. This article deals with the (semi-peripheral) constitutive nature and structural position of the rising regional powers within the BRIC Forum. Likewise, we will examine each of the Forum's summits and the geopolitics that has been fostered by the BRIC countries. According to our analysis, there is evidence that all members of the Forum are called to play important roles in the processes of change and transformation in the international political order of the $21^{\text {st }}$ century.
\end{abstract}

Key words: International Political System; BRIC; regional powers; geopolitics; semi-periphery.

\section{As potências regionais como protagonistas do sistema político internacional: cooperação e diálogo no Fórum BRICS}

\begin{abstract}
RESUMO
As potências regionais começaram a desempenhar papéis geoeconômicos e geopolíticos destacados no Sistema de Relações Internacionais desde os anos 2000, quando se fez evidente que haviam iniciado um processo de ascensão na pirâmide do poder político mundial. Desde então, estas potências regionais começaram a se aproximar, convergir e cooperar, abrindo caminho para a formação de um Fórum Internacional: o BRICS. Rapidamente este Fórum se converteu em um ator internacional coletivo de primeira ordem, juntamente com o G-20 e em competência com o G-7. Este artigo aborda a natureza constitutiva (semiperiférica) e a posição estrutural de potências regionais emergentes dos Estados que constituem o Fórum BRICS. Assim mesmo, analisam-se cada uma de suas cúpulas e a geopolítica que implementam conjuntamente. Segundo nossa análise, tudo indica que os integrantes do fórum estão convocados a serem protagonistas no processo de mudança e transformação da ordem política internacional no século XXI.
\end{abstract}

Palavras-chave: Sistema Político Internacional; BRICS; potências regionais; geopolítica; semiperiferia.

\section{REFERENCIA NORMALIZADA}

Morales Ruvalcaba, Daniel Efrén; Rocha Valencia, Alberto, y Vargas García, Elizabeth (2013) "Las potencias regionales como protagonistas del sistema político internacional: cooperación y diálogo en el Foro BRICS”. Geopolítica(s). Revista de estudios sobre espacio y poder, vol. 4, núm. 2, 237-261.

SUMARIO: Introducción. 1. Rol de los Estados semiperiféricos en la mutación del orden internacional. 2. Potencias regionales como actores destacados en el sistema político internacional. 3. Cooperación y diálogo entre las potencias regionales en el Foro BRICS. 3.1. Primera Cumbre, Ekaterimburgo 2009: constitución del Foro de diálogo y cooperación. 3.2. Segunda Cumbre, Brasilia 2010: establecimiento del entramado multilateral del BRIC. 3.3. Tercera Cumbre, Sanya 2011: emplazamiento de la cooperación intra-BRICS y primera proyección como actor relevante a nivel global. 3.4. Cuarta Cumbre, Nueva Delhi 2012: reforza- 
miento como actor económico y político a nivel global. 3.5. Quinta Cumbre, Durban 2013: despliegue de los BRICS. 4. Los BRICS y la geopolítica de la "cruz del Sur". Conclusión. Bibliografía.

\section{Introducción}

La creciente importancia política y económica de las llamadas "potencias emergentes" (Gabas y Losch, 2009) ha motivado a muy diversos analistas -igual que a nosotros - a su estudio. Uno de los análisis más destacados al respecto correspondió a Jim O’Neill, economista británico y uno de los directivos del grupo Goldman Sachs, quien en 2001 encontró que el crecimiento del producto interno bruto (PIB) de Brasil, Rusia, India y China había excedido al del G7 y que esa tendencia se mantendría durante toda la década, trastocando con ello importantes equilibrios de la geoeconomía global. Jim O'Neill - en una chispa de originalidad y otro tanto de agudeza mercadológica - decidió nombrar al conjunto integrado por Brasil, Rusia, India y China como los BRIC (O'Neill, 2001).

Bajo esta primera acepción del término BRIC, resultaba muy difícil encontrar un denominador común que fuera más allá del vertiginoso crecimiento económico. Sin embargo, una enorme voluntad política cristalizaría lo que se desestimaba posible: la creación del Foro BRICS.

Ante la importancia de esta nueva cuestión en la política internacional, nosotros emprendimos una investigación que nos llevó a comprender que los Estados agrupados bajo el acrónimo BRIC y, después, BRICS, eran un grupo que reunía características comunes bien marcadas: Estados semiperiféricos destacados, potencias regionales (no medias ni mundiales), potencias regionales mediadoras y ascendentes, potencias regionales impulsoras de procesos de integración regional en sus regiones de pertenencia, entre otras. En consecuencia, rápidamente pudimos ver que estas potencias regionales se habían transformado en protagonistas internacionales y globales y, por lo tanto, se habían colocado como competidores - ¿rivales?- de las potencias mundiales agrupadas en el G-7; además, habían emprendido el cuestionamiento del orden y la estructura del sistema político internacional. Esto quedó muy claro cuando estas potencias regionales fundaron el Foro BRICS. Bien entendido, las potencias regionales se han propuesto escalar la jerarquía de poder internacional, pasar al centro de la economía mundial e instalarse como nuevos poderes estatales mundiales. Pero, este es un proceso histórico que solamente se encuentra en las primeras etapas de su desenvolvimiento y, por lo tanto, todavía falta mucho por acontecer y ver. Nuestros análisis buscan dar cuenta de este momento histórico, siendo algunos de los primeros productos de investigación: "El sistema político internacional de Post-guerra Fría y el rol de las potencias regionales-mediadoras. Los casos de Brasil y México" (Rocha Valencia y Morales Ruvalcaba, 2008); "Potencias medias y potencias regionales en el sistema político internacional de Guerra Fría y Posguerra Fría. Propuesta de dos modelos teóricos" (Rocha Valencia 
y Morales Ruvalcaba, 2011), "El Foro BRICS como actor clave en el sistema internacional de Posguerra Fría" (Morales Ruvalcaba, Rocha Valencia y Vargas García, 2012) e "Inside the BRIC: analysis of the semiperipheral character of Brazil, Russia, India and China" (Morales Ruvalcaba, 2013). Para nosotros, las potencias regionales están en una larga ruta: en un inicio fueron Estados periféricos grandes, después se convirtieron en Estados semiperiféricos destacados, en un paso siguiente se transformaron en potencias regionales y luego emprendieron su ascenso en la jerarquía de poder internacional. Esto último ocurrió cuando se agruparon en el Foro BRIC, que poco después se amplió a BRICS. De esta manera, no solamente se forma un foro internacional, sino que se constituye un nuevo actor colectivo internacional muy dinámico.

Desde 2009, el Foro BRICS ha celebrado cinco Cumbres presidenciales, diversas reuniones de ministeriales (de Relaciones Exteriores, de Finanzas, Comercio, Agricultura, Salud, etc.), encuentros de representantes de gobiernos locales y de otros actores de la sociedad civil. Esto nos remite a una acepción del término BRICS: la de una organización intergubernamental informal con reuniones periódicas, objetivos compartidos y una agenda de trabajo común. En dicho tenor, cabe preguntar: ¿en qué radica la disposición política de Brasil, Rusia, India, China y Sudáfrica para cooperar?, ¿por qué Estados tan aparentemente disímiles dialogan de manera sostenida y cada vez más amplia? Hasta el primer semestre de 2013, ¿cuáles pueden ser enunciados como los principales logros alcanzados a través de las Cumbres BRICS?

Este trabajo es un eslabón intermedio entre los primeros estudios sobre potencias regionales y el nuevo proyecto de investigación que estamos implementando sobre potencias mundiales, potencias medias, potencias regionales y potencias subregionales en el sistema político internacional de Posguerra Fría. Por eso, en este trabajo, abordaremos cuestiones como el rol de los Estados semiperiféricos en la mutación del orden internacional, las potencias regionales como actores destacados de la semiperiferia, cooperación y diálogo entre estas potencias en el seno del Foro BRICS y, finalmente, el BRICS y la "geopolítica de la cruz del Sur".

\section{Rol de los Estados semiperiféricos en la mutación del orden internacional}

Nuestro punto de partida es que Brasil, India, Sudáfrica, China y Rusia, a pesar de sus avances económicos y sociales, son Estados pertenecientes a la semiperiferia de la economía-mundo y comparten dicha naturaleza. Pero, ¿en qué consiste la semiperiferia?, ¿cúales son las características de dicha zona?, ¿qué especificidades muestran los Estados semiperifericos?

Desde la teoría de análisis de los sistemas mundiales se observa al sistemamundo moderno organizado en una economía-mundo de tres áreas económicas y un sistema interestatal-internacional. Se entiende que la economía-mundo y el sistema 
interestatal-internacional interactúan recíprocamente, aunque la primera determina relativamente la segunda. Para Immanuel Wallerstein, dicha división "no es meramente funcional —es decir, ocupacional- sino geográfica" (Wallerstein, 2003a: 492). Bien entendido, las áreas que se encuentran en el sistema-mundo no son sólo un constructo teórico para comprender la división internacional del trabajo sino que son áreas geográficas existentes, reales, históricamente construidas y espacialmente establecidas. Como lo explica David Harvey, estas áreas "son perpetuamente reproducidas, sostenidas, socavadas y reconfiguradas por los procesos políticoeconómicos y socioecológicos que tienen lugar en el presente" (Harvey, 2000: 98). Esto indica que los espacios no son inherentes a un área económica, sino que son los procesos "los que estructuran el espacio" (Taylor y Flint, 2002: 21) de manera incesante y perpetua.

Las áreas de la economía-mundo son centro, semiperiferia y periferia. De las tres áreas, la semiperiferia es quizá la más complicada de precisar. La semiperiferia se entiende como "un número significativo de Estados que parecen estar permanentemente en una posición intermedia entre la «madurez» y el «retraso», como dirían los teóricos de la modernización, o entre el «centro» y la «periferia», como dirían los teóricos de la dependencia" (Arrighi y Drangel, 1986: 9). Así, el concepto de semiperiferia viene a ser una categoría analítica impresindible para cubrir el dicotómico vacío teórico existente en el modelo centro-periferia.

La semiperiferia - como la zona más dinámica de tránsito, interconexión y flujo en la economía-mundo- está conformada por procesos centrales y por procesos periféricos, dando lugar a una combinación específica de procesos que han sido llamados como llamados intermedios. Así, de manera amplia, los Estados que se han posicionado en dicha área económica han sido llamados semiperiféricos y presentan las siguientes características: a) ocupan una posición (estructural, funcional y geográfica) entre el centro y la periferia, por eso, para Immanuel Wallerstein, son Estados intermedios; b) sus economías correspondientes se encuentran en vías de industrialización; c) sus aparatos estatales están en proceso de modernización; d) muestran alta desigualdad socio-económica y fuerte disparidad en sus regiones internas; e) proyectan geoculturas atractivas e influyentes pero que distan de ser dominantes; f) contienen fuerzas y dinámicas sociales profundamente discordantes; g) son actores importantes - mas no directores - en la gobernanza global; y, h) conservan el más alto potencial transformador del sistema internacional.

Ciertamente los Estados semiperiféricos no tienen la posibilidad de competir política, económica y militarmente con los Estados centrales. Por eso, son considerados Estados intermedios entre los Estados centrales que son fuertes y los Estados periféricos que son débiles. Sin embargo, esto no opaca ni pone en segundo plano la importancia de la semiperiferia en sistema internacional ya que, en cuanto al potencial de transformación sistémico, es ésta el área más relevante y crucial. Para comprender y dimensionar dicho potencial, es preciso indagar en la génesis de la semiperiferia. 
Immanuel Wallerstein brinda dos respuestas para el surgimiento de la semiperiferia en su primer tomo de "El moderno sistema mundial": una, que puede tratarse de zonas que "eran áreas centrales en versiones anteriores de una cierta economíamundo" (Wallerstein, 2003a: 492), es decir, "antiguas áreas centrales en evolución hacia estructuras periféricas" (Wallerstein, 2003a: 144); o, bien, pudieron ser "áreas periféricas, promocionadas más adelante, por así decirlo, como resultado de la geopolítica cambiante de la economía-mundo en expansión" (Wallerstein, 2003a: 492). Lo anterior es continuado y ampliado por Immanuel Wallerstein en el segundo tomo de la misma obra donde su objetivo es estudiar el proceso de consolidación del sistema mundial. Aquí, se explica que las variaciones en la fuerza económica relativa "pueden ser consideradas - y de hecho lo son muy a menudo- como una especie de «movilidad» ascendente o descendente del Estado como entidad, movimiento que se mide en relación con los demás Estados dentro del marco del sistema interestatal" (Wallerstein, 2003b: 247). Así, "la semiperiferia no sería una posición fija, sino más bien un punto de ascenso/descenso de los países" (Domingues, 2012: 18), ya que "no hay procesos semiperiféricos; más bien, el término de «semiperiferia» se aplica directamente a zonas, regiones o Estados en los que no predominan ni los procesos de centro ni los de periferia" (Taylor y Flint, 2002: 22).

Aquí se confirman entonces dos fuerzas polarizantes que arrastran a las zonas semiperiféricas en distintas direcciones: por un lado, dinámicas de periferización que subordinan dichas zonas a las necesidades de los Estados centrales; y, por otro lado, dinámicas de centralización que buscan hacerlas transitar hacia las áreas económicas del centro. Estas dinámicas, finalmente, se sintetizan en un punto intermedio del continuo jerárquico de poder internacional y, en algunos casos, emprenden la vía del ascenso. Evidentemente, las posiciones estructurales en el sistema-mundo son históricas y, aunque pueden lograrse cambios significativos de ascenso/descenso, estos sólo son observados en los tiempos de moyenne durée y longue durée.

Así, la semiperiferia se ve determinada e influida por procesos centrales, de la misma forma en que se ve afectada e intervenida por procesos periféricos; es por ello que algunos Estados semiperiféricos - y sólo unos pocos- pueden ser impulsados hacia el centro, o bien pueden ser frenados y arrastrados hacia la periferia. Todo dependerá de la calidad de sus gobiernos nacionales, el acierto de sus políticas públicas, la importancia de sus modelos de desarrollo y la buena conducción de sus políticas exteriores. Como apuntan algunos, "las oportunidades de cambio se producen en los períodos de recesión, pero son muy limitadas, porque no toda la semiperiferia puede convertirse en centro" (Taylor y Flint, 2002: 22). Es en los cambios de fase de los ciclos de Kondratieff cuando se presentan más claramente dichos períodos (Kondratieff, 1946) de cambio estructural y mutación en el orden internacional.

Ahora bien, entre los Estados que conforman la semiperiferia solamente algunos, por las razones ya esgrimidas, han emprendido el ascenso estructural y, gracias a 
ello, manifiestan una creciente preponderancia geopolítica y geoeconómica en sus respectivas regiones. Éstos han sido denominados "como «jugadores estratégicos» y potencias regionales (Zbigniew Brzezinski); «Estados industriales en proceso de modernización» (Joseph S. Nye); los BRIC «una fuerza dominante» en la economía del mundo (Dominic Wilson y Roopa Purushothamn); «nuevos jugadores globales» (NIC); «potencias regionales» (Detlef Nolte y Daniel Flemes); «países elefantes» y «Estados escaladores» (Robert Fossaert); «potencias emergentes del Sur» (Susanne Gratius); «potencias intermedias» (Regina Soares de Lima); «países intermedios» (Ricardo Ubiraci Sennes); «Estados grandes de la periferia» (Samuel Pinheiro Guimarães), «poderes medios emergentes» o de «segunda generación» (David Dewitt y Ryerson Christie) y "poderes regionales» (Brantly Womack)" (Rocha Valencia y Morales Ruvalcaba, 2011: 108). Este fue el estado de la cuestión que quedó plasmado en el libro "Potencias medias y potencias regionales en el sistema político internacional de Guerra Fría y Posguerra Fría. Propuesta de dos modelos teóricos". Desde nuestro punto de vista, la categoría que mejor describe a dichos Estados semiperiféricos destacados es la de potencias regionales.

\section{Potencias regionales como actores destacados en el sistema político interna- cional}

Para nosotros, las potencias regionales no son potencias mundiales ni potencias medias. Las potencias mundiales son Estados desarrollados que cuentan con las mayores capacidades materiales, semimateriales e inmateriales. Esto significa que disponen de bases territoriales y poblacionales amplias, representan a los mercados comerciales y financieros más grandes del planeta (como dato estadístico significativo, todas tienen un PIB superior a US\$1.5 billones según cifras del Banco Mundial para el año 2011) y cuentan con los ejércitos más poderos y modernos del mundo, además de que sus individuos gozan de altos ingresos per cápita y sus niveles de bienestar (educación, salud, investigación y desarrollo) son muy elevados. Así, las potencias mundiales "sobresalen de todo el grupo de Estados centrales porque cuentan con capacidades para desplegar proyecciones geopolíticas, geoeconómicas y geoestratégicas de carácter mundial e internacionales" (Rocha Valencia y Morales Ruvalcaba, 2010: 258) y logran obtener, por lo general, resultados favorables en disputas militares, diplomáticas e incluso comerciales. Casos concretos de potencias mundiales son: Estados Unidos, Japón, Alemania, Francia, Reino Unido, Canadá e Italia, integrantes del Grupo de los 7 (G7). Todas ellas son potencias de primer orden y trabajan codo con codo en la definición del orden internacional, liderando y estableciendo directrices de la gobernanza mundial. Del conjunto de Estados centrales hay uno que logra destacar por su preponderancia productiva, financiera y militar durante lapsos de tiempo relativamente amplios: este Estado se 
convierte en hegemónico; durante el siglo XX y la primera década del siglo XXI ha sido Estados Unidos.

Por su parte, las potencias medias son igualmente Estados "desarrollados, modernos, democráticos y con una diplomacia muy especializada de alcance global" (Rocha Valencia y Morales Ruvalcaba, 2010: 261). Las potencias medias detentan importantes niveles capacidades semimateriales e inmateriales, esto es, ingresos per cápita elevados, bienestar socio-económico y alto desarrollo, pero a diferencia de las potencias mundiales, cuentan con capacidades materiales relativamente más limitadas ya el tamaño de sus economías es significativamente menor (de acuerdo con las cifras del Banco Mundial para el año 2011, todas tienen un PIB menor a US $\$ 1.5$ billones), sus bases poblacionales y territoriales son relativamente reducidas, su comercio y reservas internacionales no son de primera importancia a nivel mundial y sus ejércitos son menores, restricciones que posicionan a estas potencias estructural y jerárquicamente por debajo de las potencias mundiales. Casos de potencias medias se encuentran en Australia, España, Corea del Sur, Holanda, Noruega, Suecia, Bélgica, Suiza, Dinamarca e Israel. En su comportamiento internacional se observa que las potencias medias "se inclinan por impulsar la cooperación internacional, defender el derecho internacional, fortalecer las instituciones internacionales y preservar la paz" (Rocha Valencia y Morales Ruvalcaba, 2010: 263), aunque bajo el interés de su propia preservación. Así, las potencias medias pueden ser calificadas de coadyuvadoras (Rocha Valencia y Morales Ruvalcaba, 2008: 48) ya que cooperan con las potencias mundiales en la coordinación del crecimiento económico entre los países capitalistas avanzados.

A diferencia de las potencias mundiales y las potencias medias, las potencias regionales carecen de capacidades no-materiales o semi-materiales (niveles de bienestar y desarrollo socioeconómico medio) e inmateriales (atractivo cultural e ideológico), pero cuentan con elocuentes y muy importantes capacidades materiales (economías grandes y dinámicas, mercados nacionales amplios, vastas extensiones territoriales, ejércitos numerosos, niveles de intercambio comercial y de reservas internacionales importantes). Esto les permite, entre otros aspectos, demarcar geopolíticamente una región, trazar proyectos de articulación de infraestructura, esbozar idearios políticos en espacios regionales, ejercer liderazgo y eventualmente supremacía entre sus vecinos, participar activamente en elaboración de la agenda regional, impulsar sistemas de integración y crear instituciones de gobernanza regional, desplegar una política exterior proactiva en defensa de los intereses regionales y cooperativa con otras potencias regionales. Ejemplos de potencias regionales, tal como se ilustra en la Figura 1, son China, Brasil, Rusia, México, Arabia Saudita, Turquía, India, Sudáfrica y, de manera menos evidente, Argentina y Polonia. 
Figura 1. Índice de Poder Mundial para potencias regionales, 19902011

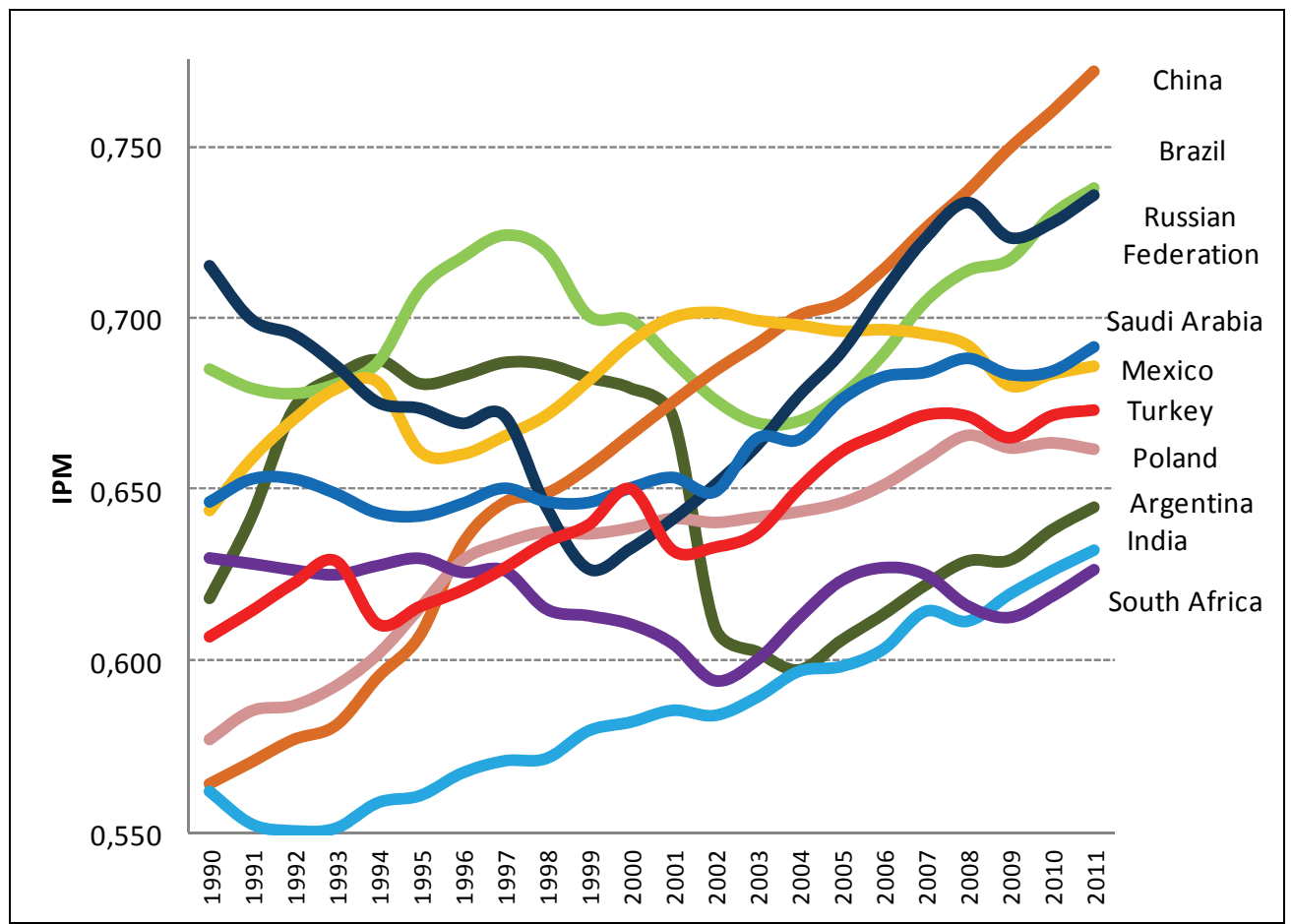

Elaboración propia: Grupo de Investigación sobre Política Mundial. Fuente: Morales Ruvalcaba, Registro Público del Derecho de Autor 2013

Actualmente, las potencias regionales no son aún potencias de primer orden, tal como se puede comprobar en la Figura 2 que muestra el posicionamiento estructural en el sistema internacional de distintos países a través del Índice de Poder Mundial ${ }^{1}$ (IPM). No obstante, aunque las potencias regionales se encuentran en términos

${ }^{1}$ El IPM fue publicado por primera vez en Potencias medias y potencias regionales en el sistema político internacional de Guerra Fría y Posguerra Fría. Propuesta de dos modelos teóricos (Rocha Valencia y Morales Ruvalcaba, 2011: 109-151). Con su divulgación, el IPM ha sido sujeto de diversas críticas y observaciones que han contribuido a su actualización y mejoramiento. Así, respetando la metodología originalmente trazada, el IPM se integra ahora a partir de un Índice de Capacidades Materiales (ICM) compuesto por seis índices simples (Índice del PIB, Índice Territorial, Índice de Población, Índice de Defensa, Índice Comercial e Índice de Reservas) y el Índice de Capacidades Semi-Materiales (ICSM), igualmente compuesto por otros seis índices simples (Índice del PIB per cápita, Índice de Consumo per cápita, un Índice de Electricidad per cápita, Índice de Educación, Índice de Salud e Índice de Investigación y Desarrollo). No obstante, dejamos aquí constancia de que queda por analizar y sistematizar un conjunto de indicadores que puedan reflejar las capacidades inmateriales de un Estado-nación. 
estructurales por debajo de las potencias mundiales y cerca de las potencias medias, sus capacidades les permiten desempeñar roles de mediación entre el centro y la periferia (Rocha Valencia y Morales Ruvalcaba, 2008), detentar significativas posibilidades de coerción y liderazgo con sus vecinos regionales y proyectarse como potencias globales virtuales.

Figura 2. Índice de Poder Mundial para categorías de potencias, 19902011

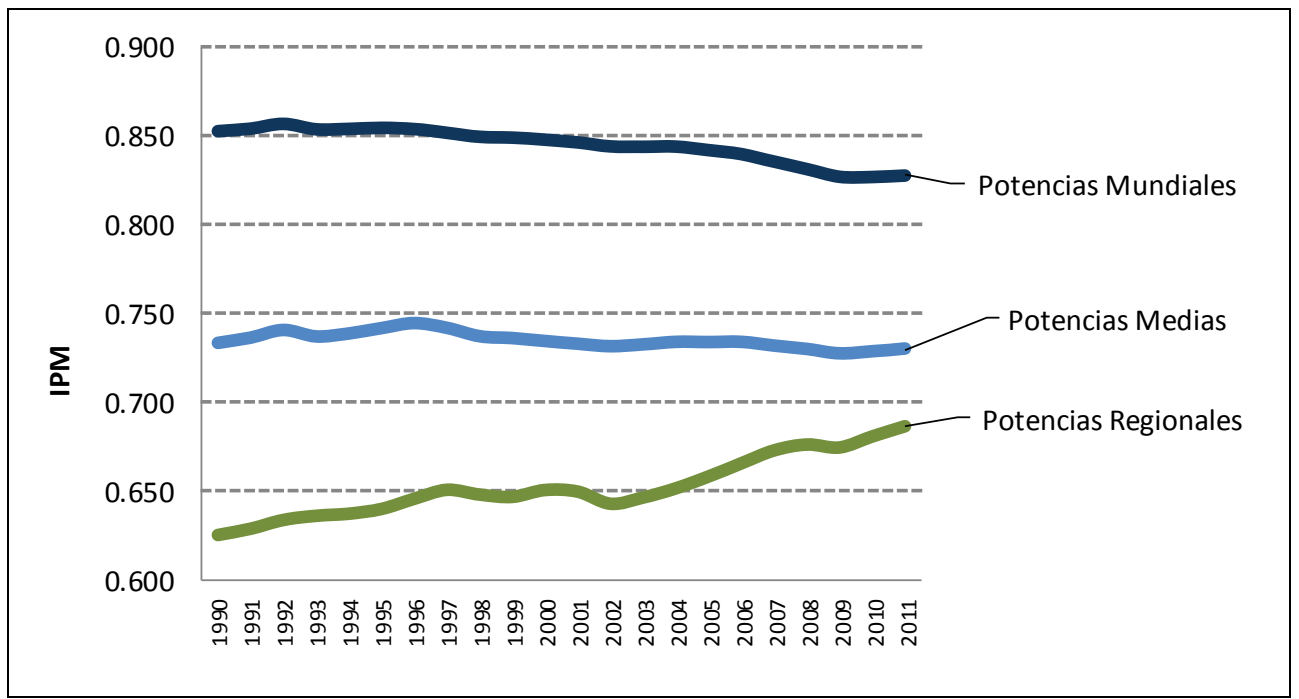

Elaboración propia: Grupo de Investigación sobre Política Mundial. Fuente: Morales Ruvalcaba, Registro Público del Derecho de Autor 2013.

Hay que destacar que, dentro conjunto de las potencias regionales, un grupo de ellas como China, Rusia, Brasil, India y Sudáfrica, países aparentemente tan disímiles, muestran una amplia disposición para dialogar y cooperar de manera sostenida y creciente; lo que no inhibe, en absoluto, la competencia entre ellas. Desde nuestro punto de vista, su propensión a la colaboración no surge de una convergencia de identidades y valores, sino de la coincidencia de dichos Estados en la semiperiferia del sistema mundial y de la convergencia de intereses a partir de sus procesos de escalada en la estructura internacional (tal como lo ilustra la Figura 2). Esta proximidad entre las potencias regionales que conforman el BRICS, ha sido estimulada aún más por la crisis económico-financiera de 2008/2009 y el paulatino ocaso de la hegemonía de Estados Unidos. 


\section{Cooperación y diálogo entre las potencias regionales en el Foro BRICS}

En el contexto de las aperturas del $61^{\circ}, 62^{\circ}$ y $63^{\circ}$ períodos de sesiones de la Asamblea General de las Naciones Unidas (AGNU) -en 2006, 2007 y 2008, respectivamente- se dieron los primeros acercamientos entre los Ministros de Relaciones Exteriores de Brasil, Rusia, India y China. Después de Nueva York, transcurrirían un par de años para que hubiera otra aproximación entre autoridades del futuro BRICS.

No será sino hasta el 16 de mayo de 2008 en Ekaterimburgo, que los Ministros de Relaciones Exteriores se reencontrarían, pero ahora en el marco de una reunión ad hoc. En el comunicado final de dicho evento, los Cancilleres se manifestaron por la construcción de un sistema internacional multilateral y más democrático (especialmente en torno a la seguridad internacional) y destacaron la cooperación SurSur como un importante elemento en los esfuerzos para alcanzar el desarrollo. El trabajo de los Cancilleres alcanzaría su más alto respaldo en la reunión informal realizada entre los jefes de Estado de Brasil, Rusia, India y China al margen de la XXXIV Cumbre del G7/G8 en Tōyako el 8 julio de 2008.

A partir de estos primeros encuentros comenzará a desenvolverse una progresiva y ambiciosa agenda entre los futuros socios primero del BRIC y, después, BRICS (con la incorporación de Sudáfrica). Como evidencia de ello, pueden mencionarse la I Reunión de Ministros de Economía y Finanzas del 7 de noviembre de 2008 en São Paulo, el I Seminario de Think Tanks acaecido el 17-18 de noviembre de 2008 en Moscú, la I Reunión de Altos Funcionarios Responsables en temas de Seguridad celebrada del 28 al 30 de mayo de 2009 también en Moscú y el I Foro Académico -o Pre-BRIC Summit Preparatory Meeting- realizado el 13-14 de mayo de 2009 en Nueva Delhi. Todos estos esfuerzos derivarían en la I Cumbre BRIC.

\subsection{Primera Cumbre, Ekaterimburgo 2009: constitución del Foro de diálogo y cooperación}

Los resultados de la Cumbre de Ekaterimburgo, celebrada el 16 de junio, quedarán plasmados en un documento estructurado en 16 puntos. En una síntesis, se puede mencionar como puntos más destacados del Comunicado Conjunto (BRIC, 2009):

1. Enfatizar el papel desempeñado por el G20 para hacer frente a la crisis financiera.

3. Compromiso por la reforma de las instituciones internacionales, en pro de las economías emergentes.

8. Apoyo a la cooperación energética. 
10. Compromiso por aumentar la cooperación en áreas socialmente vitales, provisión de ayuda humanitaria y reducción de riesgos de desastres naturales.

11. Compromiso por la investigación y el desarrollo de tecnologías avanzadas.

12. Apoyo a un orden mundial multipolar más democrático y justo basado en el derecho internacional.

14. Compromiso por la diplomacia multilateral y la reforma de la ONU.

15. Acuerdo para que el diálogo y la cooperación entre los BRIC coadyuven a la construcción de un mundo armonioso de paz duradera y prosperidad común.

\subsection{Segunda Cumbre, Brasilia 2010: establecimiento del entramado multilateral del BRIC}

En el año 2010, la crisis económico-financiera global todavía no ha sido completamente resuelta. Y aunque el Foro BRIC ya había celebrado su primera cumbre con la clara intención de coordinar posturas en organizaciones internacionales y demandar mayor participación en la gestión de la crisis, pocos hacían votos a favor de la viabilidad del BRIC. A pesar del escepticismo respecto del futuro del bloque, a partir de Ekaterimburgo se continuó con la intensa cooperación multisectorial, realizando para ello otra reunión informal entre los Ministros de Relaciones Exteriores el 24 de septiembre de 2009 en Nueva York (64 período de sesiones de la AGNU), la I Reunión de Ministros de Hacienda al margen de la Cumbre del G20 el 24-25 de septiembre de 2009 en Pittsburgh, la I Reunión de Jefes de Instituciones Estadísticas Nacionales el 22 de febrero de 2010 en Nueva York, el I Programa de intercambio de Magistrados y Jueces del 1 al 12 de marzo de 2010 en Brasilia, I Reunión de Ministros de Agricultura el 26 de marzo de 2010 en Moscú, el I Encuentro de los Representantes de Bancos de Desarrollo el 13 de abril de 2010 en Río de Janeiro - y donde trascendió la firma de un Memorándum de Entendimiento entre las partes con miras a la fomento de proyectos de infraestructura-, el II Seminario Think Tanks el 14-15 de abril de 2010 en Brasilia y la II Reunión de Altos Funcionarios Responsables en temas de Seguridad el 15 de abril de 2010, por mencionar los más destacados.

Con esta dinámica, es posible apuntar que el objetivo de la II Cumbre - celebrada en Brasilia el 15 de abril de 2010 - fue dotar al BRIC de contenido político y de mayor credibilidad internacional. Así, los acuerdos de la Cumbre de Brasilia quedaron plasmados en una Declaración Conjunta de 33 puntos (BRIC, 2010), de entre los cuales se pueden subrayar: 
1. Se comparte la percepción de que el mundo está pasando por cambios rápidos e importantes, que realzan la necesidad de transformaciones en la gobernanza global.

2. Se subraya el apoyo por un orden mundial multipolar, equitativo y democrático.

4. Se comprometen con la diplomacia multilateral y la reforma de ONU.

10. Creen en que el mundo necesita de una arquitectura financiera reformada y un sistema monetario más estable, previsible y diversificado.

11. Se comprometen en alcanzar una conclusión ambiciosa para las reformas de las instituciones de Bretton Woods (especialmente, en lo referente al poder de voto).

12. Estudian las posibilidades de cooperación monetaria.

18. Instan a la comunidad internacional a hacer todos los esfuerzos necesarios para combatir la pobreza, la exclusión y la desigualdad, teniendo en cuenta las necesidades especiales de los países menos desarrollados.

25. Afirman la importancia de incentivar el diálogo entre civilizaciones, culturas, religiones y pueblos.

Evidentemente la Cumbre de Brasil y la Declaración Conjunta resultante de ésta, son la culminación de un trabajo multisectorial arduo y profundo realizado a lo largo del período 2009-2010. Con esto el BRIC — aún sin la participación de Sudáfrica- adquiere una dinámica propia.

\subsection{Tercera Cumbre, Sanya 2011: emplazamiento de la cooperación intra-BRICS y} primera proyección como actor relevante a nivel global

Así como los años 2008 y 2009 pasaron a la historia la crisis económico-financiera global, el año 2011 quedó para la posteridad por la llamada "Primavera Árabe", acontecimiento que ha sido comparado por su trascendencia con la caída del Muro de Berlín. Sin embargo, es preciso subrayar que los acontecimientos del 2011 se encuentran intrínsecamente eslabonados a las crisis de los años anteriores: las revoluciones democráticas árabes son simultáneamente causa y efecto de profundas transformaciones en el sistema-mundo moderno y en el orden internacional de Posguerra Fría (López Almejo y Morales Ruvalcaba, 2011).

Ahora bien, en la resolución de los distintos conflictos, las potencias mundiales y las potencias medias comienzan a tomar un papel protagónico a través del Consejo de Seguridad de Naciones Unidas y la OTAN. Ante sus acciones, los BRICS (incluida Sudáfrica) buscarán coordinarse y presentarse frente a los países de Medio Oriente como plausibles interlocutores. Más aún, en 2011, todos los socios BRIC participaron activamente en el Consejo de Seguridad, ya sea como miembros permanentes (China y Rusia) o bajo el estatus de miembros no permanentes: Brasil en 
el período 2010/2011 e India y Sudáfrica en 2011/2012. De esta forma, la preocupación del grupo ya no será el tema económico-financiero, sino también seguridad y prosperidad. Entre las actividades realizadas por el BRIC en el período 2010-2011 destacan la V Reunión informal entre los Ministros de Relaciones Exteriores el 24 de septiembre de 2009 en Nueva York (65 ${ }^{\circ}$ período de sesiones de la AGNU), un encuentro entre los Ministros de Finanzas al margen de la Reunión del G20 en París el 19 de febrero de 2010 y la I Reunión de Ministros de Comercio en Sanya el 13 de abril de 2010.

Así, la III Cumbre BRICS (ahora ya con la incorporación de Sudáfrica) que tiene lugar en Sanya el 14 de abril de 2010 bajo el tema "Visión amplia, prosperidad compartida, estará marcada por el esfuerzo de cohesionar al Foro y posicionarlo frente a terceros. Esto puede constatarse al estudiar la Declaración Final (BRICS 2011), ya que de sus 31 puntos destacan los siguientes:

4. Se expresan por el fortalecimiento de la cooperación, así como en la promoción de la coordinación en cuestiones internacionales y regionales de interés común.

5. Constatan el papel desempeñado por el BRICS y otros "países emergentes" al contribuir de manera significativa para la paz mundial, la seguridad y la estabilidad.

6. Plantean que el BRICS es una importante plataforma de diálogo y cooperación y se muestran abiertos al creciente compromiso y colaboración con terceros países, en especial con las economías emergentes y en vías de desarrollo, así como con organizaciones internacionales y regionales.

8. Manifiestan su fuerte compromiso con la diplomacia multilateral y Naciones Unidas y reafirman la necesidad de una reforma integral, incluido el Consejo de Seguridad.

9. Expresan su profunda preocupación por las turbulencias en Medio Oriente, comparten el principio de que el uso de la fuerza debe ser evitado.

16. Apoyan la reforma y perfeccionamiento del sistema monetario internacional, por medio de un sistema de reservas internacionales incluyente y capaz de proporcionar estabilidad y seguridad.

20. Acuerdan que acelerar el crecimiento sustentable de los países en desarrollo es uno de los principales desafíos para el mundo y, que para ello, es fundamental erradicar antes la pobreza extrema y el hambre.

27. Comparten la percepción de que la cooperación ha sido enriquecedora y se concentran en la consolidación de la cooperación intra-BRICS y en el desarrollo de una agenda propia con acciones concretas.

Para dar continuidad a este último punto y establecer una base para la cooperación futura, el BRICS formula un Plan de Acción orientado a reforzar los progra- 
mas de cooperación existentes, explorar nuevas áreas de cooperación y presentar nuevas propuestas.

Como se puede observar, en esta Cumbre de Sanya dos asuntos fueron relevantes: la inclusión de Sudáfrica como miembro de pleno derecho y el reconocimiento de que la agrupación ha ganado confianza en la articulación de posiciones a futuro en la economía mundial y en las cuestiones políticas. Que de la Declaración de Sanya se desprenda un Plan de Acción es un claro indicativo de que —además de proponer mejoras para los programas de cooperación existentes- el Foro BRICS busca participar en nuevas áreas y explorar nuevas propuestas de trabajo, que por supuesto indican una visión de largo plazo y un mayor nivel de compromiso.

\subsection{Cuarta Cumbre, Nueva Delhi 2012: reforzamiento como actor económico y político a nivel global}

Después de Sanya, explota de manera impresionante la cooperación multisectorial en el BRICS: en el período 2011-2012 serán celebradas reuniones de Ministros de Salud, de Ciudades Hermanas y gobiernos locales, de Cooperativas, de Altos Funcionarios de Ciencia y Tecnología, de Cancilleres y Vice-Ministros de Relaciones Exteriores, de Representantes de Bancos Centrales, de Ministros de Comercio, de Académicos, de Empresarios, etc. Así, mientras que el tema central de las Cumbres de Ekaterimburgo y de Brasilia fue la crisis económico-financiera y en Sanya se prestó mayor atención al tema de las revoluciones árabes y reforma de algunas instituciones multilaterales, la IV Cumbre BRICS — realizada en 29 de marzo de 2012 en Nueva Delhi- tendrá como reto continuar y profundizar la asociación entre las potencias regionales. Bajo el tema "Asociación de los BRICS para la estabilidad, seguridad y prosperidad" fue que se celebró la IV Cumbre del Foro BRICS.

Siguiendo con la mecánica del año anterior, fueron publicados una Declaración (de 50 puntos) y un Plan de Acción (BRICS, 2012). De esta IV Cumbre es posible destacar los puntos:

3. Al ser provenientes de Asia, África, Europa y América Latina, plantean que la dimensión transcontinental del BRICS gana valor y significado.

6. Consideran crucial para las economías avanzadas adoptar políticas macroeconómicas y fiscales responsables.

8. Demandan una arquitectura financiera más representativa.

9. Muestran preocupación por el lento ritmo de las reformas de las cuotas y de la gobernanza del FMI.

11. Reconocen que hay una necesidad urgente de ampliar la disponibilidad de los recursos de financiamiento al desarrollo de las economías emergentes y en desarrollo. 
13. Consideran la posibilidad del establecimiento de un nuevo Banco de Desarrollo (léase Banco BRICS).

18. Concuerdan trabajar juntos para intensificar los flujos de comercio e inversión entre sus países.

19. Reconocen la importancia vital que la estabilidad, la paz y la seguridad de Medio Oriente y el Norte de África tiene para todos.

26. Reafirman la necesidad de una amplia reforma de las Naciones Unidas, incluyendo su Consejo de Seguridad.

40. Toman nota de los sustantivos esfuerzos realizados para profundizar la cooperación intra-BRICS en numerosos sectores.

En el Plan de Acción, los mandatarios pasan revista de los distintos encuentros y reuniones sectoriales y apuntan como nuevas áreas de cooperación a ser exploradas: I) la cooperación multilateral en energía, II) evaluación académica general sobre la futura estrategia de largo plazo para el BRICS, III) Diálogo del BRICS sobre políticas para la juventud, y IV) cooperación sobre temas relacionados a la población.

\subsection{Quinta Cumbre, Durban 2013: despliegue de los BRICS}

El ciclo de Cumbres entre los BRICS se cerró en el 26-27 de marzo de 2013 en Durban. No obstante, cada país miembro ha refrendado su compromiso con el grupo y el BRICS tiene ahora abiertos más frentes de cooperación que nunca antes.

La concordancia de intereses y acciones entre Brasil, Rusia, India, China y Sudáfrica ya no se limita sólo a lo temático, sino que ahora también se proyecta en lo geográfico. Muestra de ello ha sido el tema de la V Cumbre: "BRICS y África: Cooperación para el desarrollo, integración e industrialización". Las reflexiones resultantes de la reunión entre los presidentes del Foro reflejan la complacencia con la dinámica que, desde su creación, han dotado al sistema internacional y la confianza en el horizonte de reconfiguración de las relaciones internacionales con nuevos modelos y enfoques que reconozcan los retos y oportunidades del sistema internacional.

Al igual que en Sanya y Nueva Delhi, la Cumbre de Durban dejó como constancia una Declaración Final (47 puntos) y un Plan de Acción (BRICS, 2013). Los puntos que se destacan son:

3. Se manifiestan abiertos a la cooperación con países no-miembros de los BRICS, en particular con países emergentes y en vías de desarrollo.

5. Se comprometen a respaldar el proceso de industrialización africano.

6. Reafirman su fuerte compromiso en el apoyo del crecimiento y el fomento de la estabilidad financiera. 
11. Se congratularon con sus Ministros de Finanzas y Presidentes de Bancos Centrales por el trabajo emprendido con relación al Nuevo Banco de Desarrollo y al Acuerdo Contingente de Reservas, instruyéndolos a concluir los acuerdos para su establecimiento.

12. Celebraron la puesta en marcha del "Acuerdo multilateral sobre cooperación y cofinanciamiento para el desarrollo sustentable" y del "Acuerdo multilateral sobre cofinanciamiento de infraestructura para África".

16. Coinciden, puesto que está en marcha la elección de un nuevo director para la $\mathrm{OMC}$, que dicha organización requiere de un líder que esté comprometido con el multilateralismo y que sea representante de un país en desarrollo.

20. Reafirman la necesidad de una reforma integral de Naciones Unidas, así como del Consejo de Seguridad.

En esta Declaración, los BRICS no dejaron de expresar su posición común en asuntos delicados del acontecer internacional, como es el caso de Siria (punto 26) e Irán (punto 28), mientras que en el Plan de Acción se presentaron como nuevas área de cooperación: I) seguridad informática, II) lucha antidrogas, y III) intercambios juveniles y educativos. Finalmente, se acordó que Brasil sea sede de la Cumbre BRICS en 2014 y Rusia en 2015.

Evidentemente, los temas de revuelo para la prensa fueron el Banco de Desarrollo BRICS y el Acuerdo Contingente de Reservas. Con el nuevo Banco se busca movilizar recursos para el financiamiento de los proyectos de infraestructura y desarrollo de los propios miembros BRICS, pero también de otros Estados semiperiféricos y periféricos. El objetivo del Acuerdo Contingente de Reservas sería una medida precautoria ante el agravamiento de las crisis financieras experimentadas por los Estados centrales, que aliviaría presiones de liquidez para el corto plazo y que generaría certidumbre para los Estados semiperiféricos involucrados. Con la creación de estas dos instancias, el Foro BRICS daría un paso hacia adelante en lo que se refiere a su institucionalidad como organización intergubernamental, lo que supone una alternativa -o bien un desafío - tanto para el Banco Mundial como para el Fondo Monetario Internacional, instituciones nacidas en al fin de la Segunda Guerra Mundial y que aún hoy reflejan los intereses de Estados Unidos y sus aliados del G7.

Así, a partir de una cooperación multisectorial y un entendimiento en una amplia gama de asuntos, los integrantes del Foro BRICS en particular van paulatinamente cuestionando y reconfigurando el orden internacional todavía vigente, desplegando una geopolítica nueva que llamaremos la "geopolítica de la cruz del Sur". 


\section{Los BRICS y la geopolítica de la "cruz del Sur"}

Los BRICS son los líderes del conjunto de las potencias regionales y de los Estados semiperiféricos. Este estatus está dado por su posición estructural y el poder nacional/internacional que han logrado conformar. Por ese estatus y esa posición de poder los BRICS han sentado su presencia en el sistema político internacional y en el mundo; así, la geopolítica mundial es un dominio que se presenta ante ellos como un gran desafío. Pues bien, los BRICS se han comenzado a desplazar en la vertical y la horizontal del sistema de coordenadas cartesianas del mundo: en la vertical ascienden, compiten y disputan con base en su poder en la jerarquía internacional; en la horizontal se establecen, fortalecen y cooperan con la finalidad de acrecentar su poder nacional/internacional. Estos son dos de sus movimientos geopolíticos más característicos, quedando en claro que - por medio de dichos movimientosbuscan su proyección regional, internacional y mundial. Esta es pues la geopolítica de los BRICS o geopolítica de la "cruz del Sur". Por la noción de "cruz del Sur" solamente queremos resaltar los dos movimientos geopolíticos claves que realizan las potencias regionales del BRICS: el movimiento ascendente Sur-Norte (en la vertical) y en el movimiento autoconstituyente Sur-Sur (en la horizontal).

Veamos el movimiento ascendente (Sur-Norte):

A)Los Estados miembros del Foro BRICS buscan acrecentar sus capacidades materiales, sus capacidades semi-materiales (o no-materiales) y sus capacidades inmateriales ${ }^{2}$. Disponen de capacidades materiales abundantes, aunque todavía en situación de heterogeneidad, pero remarcables. Es evidente que si logran consolidarlas y acrecentarlas, contarán con una base material fuerte capaz de impulsarlas y de sustentar su ascenso estructural.

B) Los integrantes del BRICS se han propuesto modificar su posición estructural, esto significa escalar en la "pirámide de poder internacional". Todo dependerá del trabajo que realicen los gobernantes de estos países para incrementar el poder nacional/internacional de sus Estados, es decir, sus capacidades materiales, semi-materiales e inmateriales. La posición estruc-

\footnotetext{
${ }^{2}$ Respecto de la teoría del poder nacional-internacional, nuestras elaboraciones tienen como referentes las ideas de Hans Morgenthau, Joseph Nye, Kenneth Waltz, Kepa Sodupe (2002) e Immanuel Wallerstein, lo que nos permitió una primera elaboración del poder y la formulación de un primer índice de poder mundial (IPM). Éstas nos permitieron desarrollos en términos de la medición del poder y el análisis del posicionamiento estructural de los Estados. Sin embargo, después del procesamiento de las críticas recibidas a la obra "Potencias medias y potencias regionales en el sistema político internacional de Guerra Fría y Posguerra Fría: dos modelos teóricos" (Rocha Valencia y Morales Ruvalcaba 2011), decidimos avanzar en una reformulación de la teoría del poder nacional-internacional que conduce a considerar tres tipos de capacidades: las materiales, las o semi-materiales (no-materiales) y las inmateriales. Este trabajo se encuentra en proceso de elaboración.
} 
tural puede cambiar de manera paulatina y sostenida en el medio y largo plazos si se mantiene el impulso ascendente o escalador en la pirámide de poder mundial.

C) El movimiento ascendente de los países del BRICS cuestiona el poder nacional-internacional y la posición estructural de los Estados centrales y de sus líderes, los Estados del G-7. Estamos pues ante el cuestionamiento del orden internacional existente (fundado después de la II Guerra Mundial) y su puesta en crisis (declive de la superpotencia y de las potencias mundiales). Presenciamos hoy la disputa abierta y el posible conflicto entre las potencias mundiales del G-7 y las potencias regionales (y protagonistas globales) del Foro BRICS. Por el momento, los dos actores internacionales más relevantes de la política real internacional son el Foro G-7 y el Foro BRICS.

D)El movimiento ascendente de los BRICS tiende a modificar y cambiar la estructura del sistema político internacional, en consecuencia esta estructura hace crisis. La estructura del sistema político internacional que se conformó después de la II Guerra Mundial, no desapareció completamente con el derrumbe del Muro de Berlín y el fin de la Bipolaridad. El sistema político internacional quedó muy afectado, pero se mantuvo debido a la resistencia de la superpotencia estadounidense y de las potencias mundiales que se asociaron con ella. Podemos anotar que la desarticulación del llamado bloque de países socialistas y la implosión de la Unión Soviética, fue algo así como el equivalente a la crisis de un orden regional, pero no de todo el orden internacional. Es más, luego de la crisis de ese orden regional, han seguido las crisis de otros órdenes regionales como el asiático, el latinoamericano, el africano y el de Medio Oriente. La crisis real del orden internacional de Guerra Fría solamente ahora se encuentra en pleno auge. Estados Unidos al fin se ha encontrado con un competidor serio como China, y el G-7 ya tiene en frente a los BRICS.

E) En el horizonte de corto y mediano plazos se está configurando una multipolaridad conformada por potencias mundiales en declive relativo y potencias regionales-globales en ascenso relativo. Posiblemente se llegue a la conformación de una "bi-multipolaridad" —en alusión a la "unimultipolaridad" de Samuel Huntington—, es decir una bipolaridad acotada (dos polos, Estados Unidos y China) en el contexto de una multipolaridad amplia. No es nada extraño que se haya constituido un Foro Internacional como el G-20, donde se reúnen potencias mundiales, medias y regionales. Así, entre ellas, la competencia y la cooperación podrá seguir su curso.

F) En este eje vertical las potencias regionales, hoy protagonistas globales, enfrentan el desafio de transformarse en actores globales. Las potencias regionales podrán transformarse en actores globales plenos cuando hayan logrado incrementar su poder nacional/internacional y mejorado sustanti- 
vamente sus capacidades nacionales. Entonces podrán proyectarse globalmente en términos económicos, sociales, políticos y culturales.

G)Finalmente, los socios del BRICS se han conformado como actores destacados de la política internacional, pues sin la acción y rol de dichos países ya no se puede entender ésta. Los integrantes del BRICS están en diversas organizaciones internacionales (ONU, OMC, G20, etc.) y su participación cada vez es de más peso. En ellas, además de incrementar su participación, buscan cambiar sus objetivos y alcances. Este es el campo formal de la política internacional: la lucha por la modificación y ampliación del derecho internacional, la orientación y conducción de las organizaciones internacionales y la contienda por la reforma de la ONU.

Veamos ahora el movimiento autoconstituyente (Sur-Sur):

A)Los socios del BRICS buscan establecerse como potencias regionales, para ello tratan de fortalecer sus bases geoeconómicas y geopolíticas y se han propuesto rediseñar sus modelos de desarrollo económico-social y sus políticas públicas. El problema del acrecentamiento de sus capacidades materiales (en primer lugar) y sus capacidades semi-materiales e inmateriales (en segundo lugar) está a la orden del día. Modelos de desarrollo alternativos, políticas públicas adecuadas y gobiernos responsables tendrán que conjugarse a mediano y largo plazos, por lo que la carrera es larga e intensa. Ahora, si para mejorar las capacidades materiales será necesario un trabajo intenso, para impulsar el despegue de las capacidades semimateriales e inmateriales, que vienen rezagadas, todavía hará falta una dedicación mayor y más tiempo. El atraso en bienestar social es fuerte, aunque se ha comenzado a resolver; de igual manera sucede con la calidad democrática e institucional, la identidad nacional y la proyección cultural. Ya se han comenzado a atacar estos problemas, pero estos rezagos solamente pueden ser resueltos en el medio y largo plazos.

B) Ahora bien, como lo anterior no es suficiente, a partir de su base territorial impulsan procesos de integración regional con los Estados vecinos, esto es buscan regionalizarse para disponer de mayores alcances geoeconómicos y geopolíticos y para destacar como actores regionales, lo que los conduce a mejorarse como jugadores globales. La regionalización implica contribuir a la formación de sistemas de integración regional donde jueguen el rol de catalizadores y líderes. Hasta el momento todos los miembros del Foro BRICS se encuentran en el centro de sistemas de integración regional, como el Mercado Común del Sur (MERCOSUR) y la Unión de Naciones Suramericanas (UNASUR), la Comunidad de Desarrollo de África Austral (SADC, por sus siglas en inglés), la Comunidad de Estados Independientes (CEI), la Asociación Surasiática para la Cooperación Regional 
(SAARC, por sus siglas en inglés) y la Organización de Cooperación de Shanghai (OCS), entre otros.

C) Los integrantes del BRICS buscan establecer relaciones Sur-Sur para cooperar entre sus pares del BRICS y otras potencias regionales. Esta cooperación los acerca y une, aunque también los distancia porque compiten abiertamente entre ellos. China no ha manifestado aversión alguna a buscar establecer relaciones asimétricas con sus pares del BRICS y menos cuando establece relaciones de dependencia con Estados periféricos. La manera como China y los otros miembros del Foro BRICS manejen sus relaciones de cooperación y competencia entre ellos será decisiva. Si prima la cooperación sobre la competencia podrán actuar conjuntamente, si prima la competencia sobre la cooperación tenderán a la desunión y la dispersión; en el primer caso se unirán y se fortalecerán y podrán jugar roles importantes en la transformación del mundo, en el segundo caso se dispersaran y debilitarán y se volverán secundarios en todos los cambios que se impriman en orden internacional y en el mundo.

D) Los Estados miembros del Foro BRICS también establecen relaciones SurSur para cooperar con Estados periféricos y definir zonas de influencia en diversas regiones del mundo. Las relaciones Sur-Sur conllevan a pensar en el porvenir de los Estados periféricos. Potenciar la cooperación de los BRICS con los Estados periféricos podría reforzar el movimiento hacia el cambio y transformación del mundo. Enfatizar la competencia de los BRICS con los Estados periféricos podría conducir a establecer relaciones asimétricas y de dependencia, lo que redundaría en situaciones negativas. Si los BRICS pudieran vencer las tentaciones anotadas anteriormente, entonces contarán con mayores posibilidades para avanzar hacia perspectivas innovadoras.

E) Hay en la periferia (latinoamericana, africana y asiática) un conjunto de Estados periféricos ascendentes (como Irán, Venezuela, Colombia, Indonesia, Kazajistán, Ucrania, Egipto, Filipinas, Pakistán y Nigeria), que buscan llegar a la semiperiferia: sus esfuerzos son muy importantes y podrían ser buenos aliados de los BRICS y de las otras potencias regionales. Pero no solamente estos Estados periféricos se mueven, hay otros Estados en la periferia que buscan seguir dicho movimiento. Todos estos Estados periféricos también están contribuyendo all cambio del orden internacional y del mundo.

F) Es evidente que los socios del BRICS se mueven con mucha facilidad en el eje horizontal, pues son líderes entre los Estados semiperiféricos y periféricos. Pero, este liderazgo no está todavía consolidado. Para que un Estado del BRICS sea un líder necesita no solamente consolidar sus capacidades materiales y no materiales, también requiere de capacidades inmateriales importantes. Entre las capacidades inmateriales se encuentran 
la calidad democrática, la identidad nacional (valores culturales), la diplomacia, la cooperación, los medios de comunicación, entre otras. Pero para que esto suceda, las capacidades materiales deben sustentar a las capacidades semi-materiales y estas dos corresponder con las capacidades inmateriales; además las capacidades inmateriales deberán retroalimentar a las otras dos y trazar la ruta para su desenvolvimiento. Desde nuestro punto de vista, los BRICS tienen el desafío de implementar su respectivo smart power teniendo en cuenta que Estados Unidos ha diseñado su propia concepción de éste (Armitage y Nye, 2007).

La geopolítica de la cruz del Sur es el símbolo de los dos movimientos estructurales que se encuentran impulsando las potencias regionales socios del Foro BRICS en el sistema político internacional: escalar-ascender en la vertical y constituirsedesarrollarse-fortalecerse en la horizontal. Esta geopolítica, que corresponde con una geoeconomía, es altamente cuestionadora y transformadora del sistema político internacional.

\section{Conclusión}

La crisis del sistema político internacional y el ocaso de la hegemonía estadounidense han sido aprovechados por algunas potencias regionales que se encuentran en franco y sostenido ascenso estructural desde hace ya varios años. Entre dichas potencias, las que más destacan son los llamados BRICS, ahora coaligados en un foro internacional que puede ser considerado como una organización intergubernamental.

Las potencias regionales, en general, y los socios del BRICS, en particular, han sido y siguen siendo Estados semiperiféricos. No obstante, en años recientes, estos últimos han experimentado un crecimiento económico importante: hoy son países con algunos sectores industriales competitivos, los niveles de consumos de sus sociedades nacionales se han ampliado significativamente, han acumulado importantes reservas en moneda extranjera y se han convertido en exportadores de capital. Todo esto podría haberlos puesto en tránsito hacia el centro de la economía-mundo. Del conjunto de los BRICS, China es el caso más avanzado.

Asimismo, las potencias regionales son entidades estatales ascendentes en la jerarquía de poder internacional, pero todavía no han logrado afirmarse como potencias mundiales o de primer orden. En consecuencia, siguen desplegándose en sus respectivas regiones y buscando el ascenso en todo momento para manifestarse como protagonistas globales. Lo anterior podría terminar conduciéndolas al pedestal de potencias mundiales.

El Foro BRICS está incidiendo de manera puntual en la dimensión política del sistema internacional. Los miembros de la agrupación tienen como punto de partida 
un conjunto de elementos en común que les permiten posicionarse de manera similar en la estructura internacional: todavía las potencias del BRICS se encuentran posicionadas por debajo de las potencias mundiales y de las potencias medias. La excepción, una vez más, es China, ya que en años recientes habría rebasado a las potencias medias.

El Foro BRICS se ha constituido siguiendo un proceso sumamente interesante y creativo: constitución del Foro de diálogo y cooperación; establecimiento del entramado multilateral; emplazamiento de la cooperación intra-BRICS y primera proyección como actor relevante a nivel global; reforzamiento como actor económico y político a nivel global; despliegue de los BRICS. Brasil, Rusia, India, China y Sudáfrica comparten la idea de que deberían tener cierto grado de injerencia en las decisiones de la gobernanza global y por ello demandan una revisión de las instituciones internacionales para que éstas tomen en cuenta sus intereses. Este punto es importante puesto que estas potencias regionales buscan edificar un orden mundial que refleje su poderío económico, con un impacto político. Por ello, la postura crítica del BRICS frente al status quo ha sido una constate en el sistema político internacional de Posguerra Fría.

Finalmente, anotamos la relevancia que reviste el doble movimiento geopolítico que realizan los miembros del BRICS: el movimiento ascendente (Sur-Norte) y el movimiento autoconstituyente (Sur-Sur), que hemos denominado como la geopolítica de la "cruz del Sur". Además, subrayamos ampliamente los orígenes geopolíticos sureños de todas estas potencias regionales.

\section{Bibliografía}

Armitage, Richard, y Nye, Joseph (2007) "CSIS Commission on smart power. A smarter, more secure America". Center for Strategic \& International Studies [URL: <http://csis.org/files/media/csis/pubs/071106_csissmartpowerreport.pdf>, último acceso 15 de Noviembre de 2013].

Arrighi, Giovanni, y Drangel, Jessica (1986) "The stratification of the worldeconomy: an exploration of the semiperipheral zone". Review, vol. X, núm. 1, 974.

BRIC (2009) "Comunicado conjunto dos líderes dos países BRIC". Ministério das Relações Exteriores [Puesto en línea el 16 de junio de 2009. URL: $<$ http://www.itamaraty.gov.br/temas-mais-informacoes/saiba-maisbric/documentos-emitidos-pelos-chefes-de-estado-e-de/comunicado-i-cupulabric>, último acceso el 24 de Abril de 2012].

BRIC (2010) "Declaração Conjunta". Ministério das Relações Exteriores [Puesto en línea el 15 de Abril de 2010. URL: <http://www.itamaraty.gov.br/temasmais-informacoes/saiba-mais-bric/documentos-emitidos-pelos-chefes-de-estadoe-de/comunicado-ii-cupula-bric>, último acceso el 13 de Abril de 2012]. 
BRICS (2011) "Declaração de Sanya". Ministério das Relações Exteriores [Puesto en línea el 14 de Abril de 2011. URL: <http://www.itamaraty.gov.br/sala-deimprensa/notas-a-imprensa/declaracao-de-sanya-2013-reuniao-de-lideres-dobrics-sanya-china-14-de-abril-de-2011>, último acceso el 12 de Abril de 2012].

BRICS (2012) "Declaração de Nova Delhi". Ministério das Relações Exteriores [Puesto en línea el 29 de Marzo de 2012. URL: $<$ http://www.itamaraty.gov.br/sala-de-imprensa/notas-a-imprensa/quarta-cupulados-brics-nova-delhi-29-de-marco-de-2012-parceria-dos-brics-para-aestabilidade-seguranca-e-prosperidade-declaracao-de-nova-delhi/>, último acceso el 22 de Abril de 2012].

BRICS (2013) "Declaração de e-Thekwini”. Ministério das Relações Exteriores. [Puesto en línea el 27 de Marzo de 2013. URL: $<$ http://www.itamaraty.gov.br/sala-de-imprensa/notas-a-imprensa/v-cupula-dobrics-durban-27-de-marco-de-2013-declaracao-de-ethekwini>, último acceso el 12 de Abril de 2013].

Domingues, José Maurício (2012) Desarrollo, periferia y semiperiferia en la tercera fase de la modernidad global. Buenos Aires: CLACSO.

Fondo Monetario Internacional (2013) IMF eLibrary-Data [URL: $<$ http://elibrarydata.imf.org/>, último acceso el 10 de Marzo de 2013].

Gabas, Jean Jacques, y Losch, Bruno (2009) "Fabrications and illusions of emergence", en C. Jaffrelot (ed.) The emerging states. The wellspring of a new world order. Nueva York: Columbia University Press, 13-27.

Harvey, David (2000) Espacios de esperanza. Madrid: Akal.

Kondratieff, Nikolai (1946) "Los grandes ciclos de la vida económica", en G. Haberler (ed.) Ensayos sobre el ciclo económico. México, D. F.: Fondo de Cultura Económica, 35-56.

López Almejo, José de Jesús, y Morales Ruvalcaba, Daniel Efrén (2011) "La Primavera Árabe de 2011 como un elemento motriz del nuevo orden internacional multipolar”. Debate Social (ITESO), núm. 27, 1-24.

Morales Ruvalcaba, Daniel Efrén (2013) Índice de Poder Mundial (IPM). Ed. INDAUTOR. Instituto Nacional del Derecho de Autor Patente 03-2013042511465000-01.

Morales Ruvalcaba, Daniel Efrén (2013) "Inside the BRIC: analysis of the semiperipheral character of Brazil, Russia, India and China". Austral, vol. 2, núm. 4, 141-173.

Morales Ruvalcaba, Daniel Efrén; Rocha Valencia, Alberto, y Vargas García, Elizabeth (2012) "El foro BRICS como actor clave en el sistema internacional de Posguerra Fría". Ad Universa, vol. 1, núm. 3, 15-37.

O'Neill, Jim (2001) "Building better global economic BRICs". Goldman Sachs [Puesto en línea el 30 de Noviembre de 2001. URL: $<$ http://www.goldmansachs.com/our-thinking/brics/brics-reports-pdfs/buildbetter-brics.pdf $>$, último acceso el 28 de Abril de 2012]. 
Rocha Valencia, Alberto, y Morales Ruvalcaba, Daniel Efrén (2008) "El sistema político internacional de Post-guerra Fría y el rol de las potencias regionalesmediadoras. Los casos de Brasil y México". Espiral, vol. XV, núm. 43, 23-75.

Rocha Valencia, Alberto, y Morales Ruvalcaba, Daniel Efrén (2010) "Potencias medias y potencias regionales en el Sistema Político Internacional". Geopoliti$c a(s)$, vol. 1, núm. 2, 251-279.

Rocha Valencia, Alberto, y Morales Ruvalcaba, Daniel Efrén (2011) Potencias medias y potencias regionales en el sistema político internacional de Guerra Fría y Posguerra Fría. Propuesta de dos modelos teóricos. Guadalajara: Universidad de Guadalajara.

Sodupe, Kepa (2002) La estructura de poder del Sistema Internacional. Del final de la Segunda Guerra Mundial a la Posguerra Fría. Madrid: Editorial Fundamentos.

Taylor, Peter J., y Flint, Colin (2002) Geografia política: Economía-mundo, Estado-nación y Localidad. Madrid: Trama Editorial.

Wallerstein, Immanuel (2003a) El moderno sistema mundial. Tomo I: la agricultura capitalista y los orígenes de la economía-mundo europea en el siglo XVI. México, D.F.: Siglo XXI.

Wallerstein, Immanuel (2003b) El moderno sistema mundial. Tomo II: El mercantilismo y la consolidación de la economía-mundo europea 1600-1750. México, D. F.: Siglo XXI. 